SLAC-PUB-10706

September 2004

\title{
High Energy Density Physics and Exotic Acceleration Concepts*
}

\author{
Robert Noble \\ Stanford Linear Accelerator Center, Stanford University, Stanford, CA 94025 \\ Thomas Katsouleas \\ University of Southern California, Los Angeles, CA 90089
}

\begin{abstract}
The reported results and discussions in the Working Group on High Energy Density Physics and Exotic Acceleration Concepts are summarized. The working group focused largely on lasergenerated proton and ion beams from solid targets, but also considered laser vacuum acceleration results, active media accelerator proposals, ferroelectric-based accelerator technology advances and beam conditioning concepts for free electron lasers. The charge to the working group was to develop a laser-based proton injector exceeding current capabilities in at least one important parameter.
\end{abstract}

Working group summary at the $11^{\text {th }}$ Advanced Accelerator Concepts Workshop, June 21-26, 2004, Stony Brook, New York.

* Work supported by Department of Energy contract DE-AC02-76SF00515. 


\section{SCOPE AND CHARGE TO THE WORKING GROUP}

This working group is concerned with the most creative and paradigm changing ideas for accelerator physics and technology. Typically these are less well developed than the topics considered in the other working groups and are often fertile ground for rich discussion and progress made in real time. This year's working group was no exception.

This working group focused largely on laser-generated proton and ion beams from solid targets, but also considered laser vacuum acceleration results, active media accelerators, ferroelectric-based accelerator technology and beam conditioning for free electron lasers. The charge to the working group was to develop a laser-based proton injector exceeding current capabilities in at least one important parameter. To this end we began with a number of reports highlighting recent progress in this area, followed this by an attempt to summarize the characteristics of laser-generated proton and ion beams and finished by examining how well such characteristics would serve selected applications such as injectors for high energy proton accelerators, sources for studying heavy ion fusion issues and high energy density physics and medical applications. These topics are summarized next followed by a summary of presentations on the other exotic accelerator concepts.

\section{LASER-DRIVEN PROTON AND ION SOURCES}

The phase velocity of plasma wakefields in underdense plasmas is too high to trap and accelerate heavy charged particles such as protons and ions. Instead the laser acceleration of protons and ions relies on the interaction of lasers with overdense plasmas such as those produced from solid targets or foils. There are in fact several mechanisms that lead to proton and ion acceleration in such a case, a fairly long history of experimental results going back to the early 1980's, and a recent history of controversy over the dominant physics. It is beyond the scope of this summary to comprehensively review this field or all the physical processes, nevertheless we mention a few salient features to set the scene for the reports to follow.

\section{Physical Mechanisms and Properties of Laser-Proton Sources}

Several mechanisms for accelerating protons and ions can occur in the front, center and backside of the solid (foil) targets. The backside mechanism is ubiquitous and occurs as follows: the laser first heats electrons to relativistic temperatures. Their expansion off the back surface creates a large positive space change force (eE $\mathrm{kT}_{\mathrm{e}} / \lambda_{\mathrm{d}}$ ) that causes a Coulomb explosion of the ions and a further acceleration of ions in the expanding ambipolar sheath that results. There are no complete theoretical models for the energy gain of the ions; however with the help of recent work by P. Mora [1] as reported by K. Mima and historical work by D. Forslund in the 1970's as reported by L. Silva, the group was able to obtain a useful prediction for the energy gain that appeared to agree well with empirical data gathered and reported by T. Lin. 
The optimized energy gain is of the order of $1-100 \mathrm{MeV}$ and for a large number of experiments the trend in peak energy can be approximated by

$$
\mathrm{W}_{\max } \approx 5 \mathrm{MeV}\left(\sqrt{ } \mathrm{I} / 10^{19} \mathrm{~W} / \mathrm{cm}^{2}\right)(\lambda / 1 \mu \mathrm{m}) \ln 2 \omega_{\mathrm{pi}} \tau_{\mathrm{p}} \sim 3-6 \mathrm{MeV} \mathrm{a}_{0},
$$

where I is the laser intensity, $\omega_{\mathrm{pi}} \tau_{\mathrm{p}}$ is the laser pulse length normalized to the ion plasma frequency, $a_{0}$ is the normalized laser amplitude or quiver velocity for an electron defined by $\mathrm{eE} / \mathrm{m} \omega \mathrm{c}$, assumed to be of order one or larger here, and the last expression corresponds to lasers in the 50-500 fs range. Laser pre-pulse, target preparation and target thickness also play roles in final energy gains obtained.

Experiments with TW - PW (0.1-1000 J) lasers produce prodigious charge of $10^{10}$ $-10^{13}$ particles $(1-1000 \mathrm{nC})$ and recently have been shown to yield very good beam quality. The LULI group in France for example has measured $\varepsilon_{N}<.004 \mathrm{~mm}$-mrad [2]. This is an order of magnitude better than conventional injectors and also is at $\mathrm{MeV}$ energies. Although the energy spread is large, the beam longitudinal phase space area (i.e., the longitudinal emittance) is small. That is, the energy spread is correlated with position from front to back and can be undone by appropriate phasing in a subsequent radio-frequency (rf) accelerator section. The LULI group has recently demonstrated that the proton beam can be extracted from the co-moving electrons with an applied magnetic field without degrading (e.g., due to space charge) beam quality over a short distance. The injection of this beam into a beamline with a chicane and rf accelerator to stretch and then rotate the longitudinal phase space to an upright (mono-energetic) ellipse appears to be the next milestone for this field. J. Fuchs reported that preliminary work in this direction began at the workshop. Once extracted, the low emittance and high charge of this source offer the possibility of a very high-brightness beam for injector applications.

\section{Reports on Laser-Proton/Ion Acceleration}

The group developed the above summary via a number of informative reports on experiments, simulations and applications. Experimental results from T. Lin examined the effect of target thickness and target conductivity on optimizing the energy gain of ions. J. Fuchs and T. Cowan gave recent results of the LULI collaboration including the beam quality characterization mentioned above. A. Ogata and later K. Mima discussed the role of the laser pre-pulse in beating the scaling law above at relatively low powers (TW). P. Bolton gave a historical perspective on laser ionization and laser intensity measurements.

On the simulation front we heard two stimulating talks by L. O. Silva and P. Messmer on the acceleration of protons in a shock propagating through the solid target. Under limited conditions it may be possible for the shock mechanism to dominate the energy gain from the backside sheath discussed above. B. Bowes gave a talk on 2-D simulations of radiative transport in laser-created plasmas. K. Mima presented a simulation study of staged laser foils to reach higher energy. W. Mori and T. Katsouleas proposed underdense and critical density ion acceleration mechanisms, respectively, with the potential to control the acceleration by tapering profiles. 
We also heard talks on the potential applications for laser-proton injectors. J. Alessi summarized current status of conventional proton/ion sources, their performance, and the requirements for physics facilities. T. Cowan reported on requirements for Heavy Ion Fusion and High Energy Density Physics research with ion beams.

\section{Conclusions on the Applicability of Laser-Proton Sources}

The birth of the proton beam in the high electric field of the exploding foil combined with the space charge neutralization by the electrons appears to be responsible for the key competitive advantage of laser-proton sources - ten times better normalized emittance and high charge and peak brightness. The beams also exceed the peak current density of conventional sources by many orders. Next we consider some possible applications for these beams.

The table below compares the parameters required for typical high energy proton accelerators and the laser-proton source. From this it is clear that while the charge per bunch is adequate from high energy lasers the rep rate is not. For a high rep rate 10 $\mathrm{Hz}$ TW laser, the average power is typically $1 \mathrm{~W}$, while the average power needed for the injector flux desired is $10 \mathrm{~kW}$. We note that this is similar to the average power required for next generation EUV lithography lasers, so there is a good possibility that commercial R\&D will push laser technology in this direction.

We next examined the application of these beams to Heavy Ion Fusion (HIF) research and High Energy Density Physics. Moderate to heavy ion beams have attractive properties for these applications because of the so-called Bragg peak in their energy deposition vs. distance curve in matter, thus enabling them to penetrate and deposit energy in a local and controlled region. However the requirements for HIF and even for studying the accelerator physics issues associated with the transport and focusing of HIF beams far exceeds the capability of any existing ion accelerator. Thus it is of interest to examine laser-ion sources as drivers for studying relevant beam and HED physics. The goal of the HIF community over the next 5 years is to obtain beams with energy density exceeding $10^{11} \mathrm{~J} / \mathrm{m}^{3}$. If such beams can be obtained in pulses of order $1 \mathrm{~ns}$, they can be used to study such new regimes of physics as "warm dense matter" (a peculiar state with the temperature of a plasma and the density of a solid where neither plasma nor condensed matter theory apply). Current laser-ion sources produce energy densities of this order but only for 10 ps. Prospects are good for higher energy lasers in the near future however to reach the required threshold.

The final application we considered was medical uses of proton beams. Here the average power of the laser sources may not be a limiting factor. The features that make the laser sources attractive here are their compact size and their high energy. The multi-MeV injection energy reduces the injector and linear accelerator to a single tabletop laser source. The National Radiological Institute in Japan for example has proposed replacing the injector and $10 \mathrm{MeV}$ RFQ accelerator for a proton/ion cancer therapy storage ring with a much smaller (i.e., tabletop) laser-plasma source and small rf stage [3]. 
Laser-Accelerated I ons as Sources for I on Injectors

\begin{tabular}{|c|c|}
\hline $\begin{array}{l}\text { Typical HE linac requirements } \\
\text { (proton) }\end{array}$ & Laser-accelerated protons \\
\hline - $10^{13}-10^{14} \mathrm{p} /$ pulse $10^{4} \mathrm{nC}$ & - $10^{10}-10^{13} \mathrm{p} / \mathrm{pulse}$ [TW to PW] \\
\hline $\begin{array}{l}\text { pulse duration: } 5-500 \mu \mathrm{s} \\
\quad \text { (bunch: <1 ns) }\end{array}$ & $\begin{array}{l}\text { - bunch duration: } 1 \text { ps at source } \\
\text { debunches to } 100 \text { ps in } \mathrm{cm}\end{array}$ \\
\hline - $\Delta \mathrm{E} / \mathrm{E}<10^{-2}$ at injection & - $\Delta \mathrm{E} / \mathrm{E} \sim 1\left(\mathrm{E}_{\max } \sim 10-50 \mathrm{MeV}\right)$ \\
\hline $\mathrm{f}_{\text {rep }}: 5-50 \mathrm{~Hz}$ & - $10 \mathrm{~Hz}-1 /$ hour [TW to PW] \\
\hline Injection E: $50 \mathrm{keV}$ & - $10 \mathrm{MeV}$ \\
\hline $\begin{array}{l}\text { mittance } \mathrm{N}: 0.05-0.5 \\
\mathrm{~mm}-\mathrm{mrad} \text { at injectic }\end{array}$ & - emittance N: $0.005 \mathrm{~mm}-\mathrm{mrad}$ \\
\hline
\end{tabular}

\section{OTHER EXOTIC CONCEPTS}

We heard a number of innovations in reports on laser acceleration, ferroelectric materials, active media and beam conditioners.

In the area of laser acceleration we heard experimental results from D. Umstadter who used a laser wakefield accelerated beam as a source beam of electrons with which to study vacuum acceleration in the nonlinear or ponderomotive regime. After the beam exited the plasma, he interacted this beam with a second laser beam and measured the deflection as a test of laser-vacuum acceleration. We also heard from $\mathrm{T}$. Plettner about the latest design of the LEAP (Laser Electron Acceleration Project) experiment at Stanford. This experiment is designed to probe the linear interaction between a laser field and an electron beam co-propagating at a slight angle. The interaction must be terminated at a point before the particle dephases from the laser. In the new design this is done with a sacrificial tape that is ionized and reflects the laser.

A. Kanareykin reported on new low-loss ferroelectric materials. There have been major advances in these materials in the past two years. These improvements are making ferroelectrics attractive for a variety of accelerator applications including electrically tuned cavities and switches for novel pulse compression concepts for achieving the pulse length in next linear collider-type power sources. K. Nakajima presented an overview of experimental possibilities with e+e- pair plasmas that could be produced by future high intensity lasers. 
L. Schachter expanded upon his plenary talk on active media accelerators. Most exciting was the presentation of plans for an experimental test of this idea at ATF in Brookhaven. The design is to accelerate electrons by about $6 \mathrm{MeV}$ in a meter scale of pumped $\mathrm{CO}_{2}$ gas and exceed the Cherenkov wake losses. G. Shvets presented some theoretical calculations on a method to obtain very high plasma fields by exploiting the nonlinear bistability of beat-wave generated plasma waves.

$\mathrm{J}$. Wurtele reported on the novel use of beamline elements to condition a beam for free electron laser light sources. The idea is to create a correlated energy profile on the beam that increased quadratically with distance from the axis. This compensates for the added path length of off-axis particles and gives all particles the same axial speed needed to stay in phase and lase. This scheme requires a storage ring. To overcome this, E. Esarey and C. Schroeder considered laser wakefield and laser Thomson scatter conditioners, respectively. However, each had a large laser power requirement. The use of a passive plasma is an interesting alternative in which the self-wake of the beam can be made to slow the beam preferentially on axis.

\section{SUMMARY}

In summary, we synthesized recent theoretical and experimental results on laserdriven proton and ion accelerators and developed a scaling law for the maximum energy in this scheme as well as the expected beam number and beam quality. Using these results to analyze applications suggests that modest advances will lead to useful application as injectors for high energy accelerators, heavy ion fusion beam physics studies and medicine. There were also a number of new ideas presented for possibly improving or controlling the acceleration of ions and protons. We also heard several other exotic accelerator reports ranging from laser-vacuum acceleration to acceleration in active media. As G. Shvets stated in his plenary talk on exotic acceleration schemes, there is still much room for innovation in all advanced accelerator areas materials, drivers and combinations thereof.

\section{REFERENCES}

1. P. Mora, Phys. Rev. Letters 90, 185002 (2003).

2. T. Cowan et al, Phys. Rev. Letters 92, 204801 (2004).

3. A. Fukumi et al, "Energetic Proton Generation from a Thin Foil Target Irradiated by an Intense Laser Pulse with a Prepulse," presented at the International Workshop on Fast Ignition and High Field Physics 2004, Kyoto, Japan, April 2529, 2004. 